\title{
Stratégies fédérales de santé publique visant à réduire au minimum l'introduction des maladies transmissibles au Canada
}

\author{
Bhatia $\mathrm{N}^{1}$, Sarwal $\mathrm{S}^{2 *}$, Robinson $\mathrm{H}^{2}$, Geduld $\mathrm{J}^{2}$, Huneault $\mathrm{F}^{2}$, Schreiner $\mathrm{H}^{2}$, Collins $\mathrm{S}^{2}$, Hickey $\mathrm{R}^{2}$ \\ ${ }^{1}$ Schulich School of Family Medicine and Dentistry, Université de Western Ontario, London (Ontario) \\ ${ }^{2}$ Direction générale de l'infrastructure de sécurité sanitaire, Agence de la santé publique du Canada, Ottawa (Ontario)
}

*Correspondance : shelly.sarwal@phac-aspc.gc.ca

\section{Résumé}

Contexte : La propagation des maladies transmissibles à l'échelle mondiale constitue une préoccupation croissante en raison de l'augmentation des voyages internationaux. Au Canada, bien que la prise en charge des maladies transmissibles par les services de santé publique consiste principalement en des interventions de première ligne, le gouvernement fédéral prend aussi des mesures pour prévenir et réduire l'introduction de ces maladies.

Objectif : Décrire le rôle joué par l'Agence de la santé publique du Canada (l'Agence) pour réduire au minimum l'introduction des maladies transmissibles, par la mise en place de mesures préventives qui s'appliquent aux voyageurs avant leur départ du Canada, ainsi que de mesures de détection précoce et de confinement rapide pour les voyageurs qui pourraient être atteints d'une maladie transmissible à leur arrivée au pays.

Interventions : L'Agence cherche à réduire au minimum l'introduction des maladies transmissibles au Canada par la publication de conseils de santé aux voyageurs qui sont fondés sur des données probantes et la mise en œuvre d'activités de sensibilisation ciblées à l'intention du public et des professionnels de la santé. En vertu de la Loi sur la mise en quarantaine et du Règlement sanitaire international (2005), l'Agence effectue également l'inspection de différents moyens de transport, notamment les avions et les navires, et collabore avec ses partenaires à la surveillance à la en faisant l'évaluation des voyageurs malades à leur arrivée au pays.

Conclusion : L'Agence joue un rôle important dans la prévention et l'atténuation de l'introduction des maladies transmissibles au Canada, en collaboration avec les cliniciens, les autorités de santé publique à tous les ordres de gouvernement et d'autres ministères fédéraux.

\section{Introduction}

Selon la Banque mondiale, les voyages internationaux ne cessent d'augmenter et, en 2011, le nombre de touristes internationaux a été estimé à plus d'un milliard (1). Or ce mouvement continu de personnes et de marchandises, qui arrivent au Canada par voie aérienne, terrestre et maritime, fait en sorte que le risque d'introduction de maladies transmissibles est toujours présent.

Au Canada, la lutte contre les maladies transmissibles relève principalement des autorités provinciales, territoriales et locales. Cependant, de nombreux ministères fédéraux jouent également un rôle dans la réduction de l'introduction des agents pathogènes pour les humains et les animaux, des toxines, des vecteurs et des réservoirs de maladies transmissibles (Tableau 1). De fait, de nombreuses lois fédérales ordonnent aux ministères de prendre des mesures à cette fin (2,3-8). À titre d'exemple, les lois et règlements administrés par l'Agence de la santé publique du Canada (l'Agence) incluent le Règlement sur 
l'importation des agents anthropopathogènes (2), la Loi sur la mise en quarantaine (3) et le Règlement sur l'eau potable des transports en commun (4).

Tableau 1 : Exemples d'activités menées par les ministères fédéraux pour réduire au minimum l'introduction des maladies transmissibles au Canada

\begin{tabular}{|c|c|c|c|}
\hline Agence de la santé publique du Canada & $\begin{array}{l}\text { Agence canadienne } \\
\text { d'inspection des } \\
\text { aliments }\end{array}$ & $\begin{array}{l}\text { Agence des } \\
\text { services } \\
\text { frontaliers du } \\
\text { Canada }\end{array}$ & $\begin{array}{l}\text { Citoyenneté et } \\
\text { Immigration } \\
\text { Canada }\end{array}$ \\
\hline 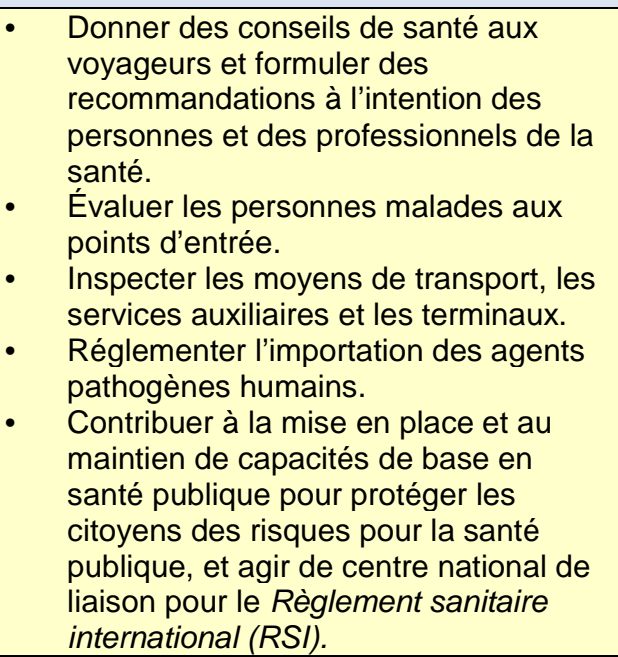 & $\begin{array}{l}\text { Réglementer } \\
\text { l'importation des } \\
\text { produits } \\
\text { alimentaires. } \\
\text { - Réglementer } \\
\text { l'importation des } \\
\text { végétaux et des } \\
\text { produits qui en sont } \\
\text { dérivés. } \\
\text { Réglementer } \\
\text { l'importation des } \\
\text { animaux vivants } \\
\text { ainsi que des } \\
\text { produits et } \\
\text { sous-produits } \\
\text { animaux. } \\
\text { Inspecter le bétail } \\
\text { aux points d'entrée. }\end{array}$ & $\begin{array}{l}\text { Procéder au } \\
\text { contrôle initial } \\
\text { des personnes, } \\
\text { des aliments, des } \\
\text { marchandises, } \\
\text { des bagages et } \\
\text { des produits } \\
\text { agricoles aux } \\
\text { points d'entrée. }\end{array}$ & $\begin{array}{l}\text { Procéder au } \\
\text { contrôle des } \\
\text { immigrants et de } \\
\text { certaines autres } \\
\text { catégories de } \\
\text { ressortissants } \\
\text { étrangers aux } \\
\text { fins du } \\
\text { dépistage de } \\
\text { certaines } \\
\text { maladies } \\
\text { infectieuses } \\
\text { avant l'arrivée } \\
\text { au Canada. }\end{array}$ \\
\hline
\end{tabular}

L'objectif du présent article est de donner un aperçu des rôles et des responsabilités de l'Agence dans la réduction de l'introduction des maladies transmissibles au Canada, par la mise en œuvre de mesures préventives s'appliquant aux voyageurs et aux moyens de transport avant leur départ du Canada, ainsi que de mesures touchant les personnes malades qui entrent au pays. Cet article ne traite pas des rôles des autorités locales, provinciales et territoriales ni de ceux des autres ministères fédéraux, ni ne cherche à en diminuer l'importance.

\section{Stratégie fédérale de santé publique}

L'Agence réduit l'introduction des maladies transmissibles au Canada par la mise en place d'activités de prévention et d'intervention ciblant les voyageurs et les moyens de transport. De l'information sur la santé des voyageurs est disponible avant que les personnes quittent le pays. Les moyens de transport internationaux sont inspectés et les risques pour la santé publique sont évalués. Les voyageurs malades sont contrôlés à leur retour au Canada. D'autres articles sur cette question examinent comment l'Agence gère la nouvelle loi qui établit un cadre d'octroi de permis fondé sur le risque pour améliorer la surveillance fédérale des agents pathogènes humains et des toxines au Canada (9) et comment l'Agence collabore, s'il y a lieu, à l'intervention rapide visant à gérer et à contenir une infection qui a été introduite au pays (10).

\section{Réduire au minimum les risques avant de quitter le Canada}

L'Agence donne des conseils de santé aux voyageurs et formule des recommandations à l'intention des voyageurs et des professionnels de la santé. Elle donne également des conseils aux exploitants de moyens de transport et procède à l'inspection des moyens de transport, des services auxiliaires (comme les cuisines de l'air) et des terminaux.

\section{Renseignements à l'intention des voyageurs}

Dans le cadre de son programme de santé des voyageurs, l'Agence publie, sur son site Web et sur le site voyage.gc.ca, des conseils de santé aux voyageurs, des fiches d'information sur des maladies liées aux 
voyages et des recommandations en matière de santé des voyageurs, selon le pays de destination (11). Les conseils de santé aux voyageurs informent le public sur les problèmes de santé, notamment les éclosions susceptibles d'être préoccupantes pour les voyageurs. Ces conseils précisent également le niveau de risque et recommandent les mesures à prendre par les voyageurs (Tableau 2).

\section{Tableau 2 : Conseils de santé aux voyageurs selon le niveau de risque}

\begin{tabular}{|c|c|c|}
\hline $\begin{array}{l}\text { Niveau de } \\
\text { risque }\end{array}$ & Explication & Exemple \\
\hline $\begin{array}{l}\text { 1. Prendre les } \\
\text { précautions } \\
\text { sanitaires } \\
\text { habituelles en } \\
\text { voyage } \\
\end{array}$ & $\begin{array}{l}\text { - Recommande aux voyageurs de prendre les précautions } \\
\text { sanitaires habituelles (p. ex. vaccinations systématiques, } \\
\text { lavage des mains, mesures de protection pour éviter les } \\
\text { piqûres de moustiques). }\end{array}$ & $\begin{array}{l}\text { Infection par le virus } \\
\text { chikungunya dans les } \\
\text { Caraïbes (en vigueur } \\
\text { en date de décembre } \\
\text { 2015) }\end{array}$ \\
\hline $\begin{array}{l}\text { 2. Prendre des } \\
\text { précautions } \\
\text { sanitaires } \\
\text { spéciales }\end{array}$ & $\begin{array}{l}\text { Recommande aux voyageurs de prendre des précautions } \\
\text { sanitaires spéciales, p. ex. recevoir des vaccins } \\
\text { supplémentaires. } \\
\text { On diffuse ce type de conseils si une région géographique } \\
\text { restreinte est touchée par une éclosion, si une nouvelle } \\
\text { maladie survient dans la région ou s'il y a eu un changement } \\
\text { de la configuration actuelle de la maladie. }\end{array}$ & $\begin{array}{l}\text { Recommandations } \\
\text { temporaires de } \\
\text { l'Organisation } \\
\text { mondiale de la Santé } \\
\text { (OMS) sur la } \\
\text { vaccination } \\
\text { antipoliomyélitique } \\
\text { (en vigueur en date } \\
\text { de décembre 2015) } \\
\end{array}$ \\
\hline $\begin{array}{l}\text { 3. Éviter tout } \\
\text { voyage non } \\
\text { essentiel }\end{array}$ & $\begin{array}{l}\text { - Il est recommandé d'éviter tout voyage non essentiel dans le } \\
\text { but de protéger la santé de la population et des voyageurs } \\
\text { canadiens. } \\
\text { - Les voyageurs sont informés des précautions particulières à } \\
\text { prendre lorsqu'ils visitent une région donnée et de ce qu'ils } \\
\text { doivent faire s'ils tombent malades durant ou après le } \\
\text { voyage. } \\
\text { On diffuse ce type de conseils si une vaste région } \\
\text { géographique est touchée par une éclosion de grande } \\
\text { envergure, si le voyageur est exposé à un risque accru et s'il } \\
\text { risque davantage de transmettre la maladie à d'autres } \\
\text { groupes, y compris à la population canadienne. }\end{array}$ & $\begin{array}{l}\text { Maladie à virus Ebola } \\
\text { en Afrique de l'Ouest } \\
\text { (en vigueur en Guinée } \\
\text { et en Sierra Leone en } \\
\text { date de décembre } \\
\text { 2015) }\end{array}$ \\
\hline $\begin{array}{l}\text { 4. Éviter tout } \\
\text { voyage }\end{array}$ & $\begin{array}{l}\text { - On recommande aux voyageurs d'éviter tout voyage dans le } \\
\text { but de protéger la santé de l'ensemble de la population du } \\
\text { Canada. } \\
\text { On diffuse ce type de conseils s'il existe un risque accru de } \\
\text { transmettre la maladie à l'ensemble de la population, } \\
\text { indépendamment des mesures prises pendant le voyage. Le } \\
\text { fait d'éviter tout voyage limitera la propagation de la maladie } \\
\text { au Canada et à l'échelle mondiale. }\end{array}$ & $\begin{array}{l}\text { Ce type de conseil n'a } \\
\text { jamais été émis }\end{array}$ \\
\hline
\end{tabular}

Des activités de sensibilisation ciblées sont également menées pour informer la population des mesures à prendre lors de rassemblements de masse. À titre d'exemple, des renseignements précis sont fournis aux Canadiens qui prévoient effectuer le Hadj, le pèlerinage musulman à La Mecque, en Arabie saoudite. En effet, pendant le Hadj, il existe un risque accru de transmission de certaines maladies transmissibles telles que la méningite à méningocoques et des infections respiratoires $(12,13)$. Depuis 2014 , divers documents, notamment des affiches et des fiches d'information, ont été rédigés et traduits en cinq langues (français, anglais, arabe, urdu et turque). Ce matériel a été distribué aux intervenants, y compris les médecins et les centres communautaires dans les communautés musulmanes, les mosquées, les écoles islamiques et les agences de voyages canadiennes autorisées à délivrer les visas pour les pèlerins du Hadj. En 2014, ces agences de voyages ont délivré quelque 3500 visas à des participants au Hadj.

Enfin, lors d'événements de santé publique précis, notamment les éclosions de syndrome respiratoire aigu sévère et de grippe $\mathrm{H} 1 \mathrm{~N} 1$, des renseignements ont été communiqués aux voyageurs dans les aéroports, au moyen d'affiches et des écrans d'affichage électronique (14). Des renseignements sur la santé des 
voyageurs sont également présentés sur le site Web voyage.gc.ca (11) ainsi que dans les médias sociaux comme Facebook et Twitter.

Renseignements à l'intention des professionnels de la santé

L'Agence fournit des services de secrétariat et un soutien épidémiologique au Comité consultatif de la médecine tropicale et de la médecine des voyages (CCMTMV), un organisme consultatif formé d'experts qui aide l'Agence dans l'élaboration des recommandations en matière de santé des voyageurs à l'intention des voyageurs et des professionnels de la santé (15). Le CCMTMV formule des recommandations fondées sur des données probantes sur la prévention et le traitement des maladies infectieuses et autres risques pour la santé auxquels les voyageurs canadiens pourraient être exposés à l'étranger. Les déclarations et les recommandations du CCMTMV sur les maladies liées aux voyages, les problèmes de santé et les populations particulières sont affichées sur le site Web de l'Agence.

\section{Réduire au minimum les risques à bord des moyens de transport}

Un moyen de transport s'entend des avions, trains, navires de croisière et traversiers (4). Le Programme du public voyageur de l'Agence collabore avec le secteur international du transport. L'Agence évalue les risques associés aux moyens de transport et aux services auxiliaires, notamment les cuisines de l'air et les terminaux, et collabore avec les exploitants afin d'éliminer tout risque pour la santé publique. Les inspections portent principalement sur l'eau potable, les aliments, les conditions d'hygiène et la lutte antivecteur. Les autres activités prévoient notamment l'échantillonnage de l'eau potable à bord des moyens de transport en vertu du Règlement sur l'eau potable des transports en commun (4), ainsi que des formations sur la manipulation des aliments et les mesures d'hygiène à l'intention du personnel de l'industrie.

Le Programme du public voyageur prévoit un programme d'inspection de la conformité pour les navires de croisière qui font escale dans les ports canadiens (16). Des inspections inattendues sont menées à bord des navires de croisière participants qui voyagent en eaux canadiennes. Le système de notation repose sur 41 éléments d'inspection, pour un maximum de 100 points. La note de passage est de 86 points sur 100. Une note inférieure ou égale à 85 n'est pas satisfaisante et requiert une nouvelle inspection dans le mois suivant. Une note insatisfaisante ne signifie toutefois pas que le public voyageur est exposé à un risque imminent pour la santé.

\section{Réduire au minimum l'introduction des maladies transmissibles}

Le Règlement sanitaire international (RSI) de 2005 définit le point d'entrée comme un point de passage pour l'entrée ou la sortie internationales des voyageurs, des moyens de transport et des marchandises; les points d'entrée incluent les aéroports, les ports maritimes et les postes frontaliers terrestres (17). Bon nombre des mesures de santé publique prises aux points d'entrée s'inscrivent dans le cadre du RSI (2005), un traité international ratifié par le Canada (18). Le but du RSI (2005) est de prévenir la propagation internationale des maladies, de s'en protéger, de la maîtriser et d'y réagir, en évitant de créer des entraves inutiles au trafic et au commerce internationaux. En vertu du RSI (2005), le Canada est tenu de prendre des mesures sanitaires aux points d'entrée pour limiter la propagation des risques pour la santé publique.

Afin de déterminer si le Canada satisfaisait aux exigences du RSI (2005) aux points d'entrée, sa capacité à détecter les risques pour la santé publique (y compris les maladies infectieuses) et à y réagir a été évaluée à cinq points d'entrée au Canada. Ces points d'entrée, qui ont été choisis en fonction du volume de voyageurs qu'ils accueillent et de leur représentation géographique, incluent l'aéroport international de Vancouver, l'aéroport international Pearson de Toronto, l'aéroport international Pierre-Elliott-Trudeau de Montréal, le terminal de navires de croisière de Metro Vancouver et le terminal de navires de croisière de Halifax. Ces points d'entrée satisfont aux exigences de l'OMS et sont donc considérés comme des points d'entrée désignés en vertu du RSI (2005). Les exigences de l'OMS relatives aux points d'entrée désignés incluent l'affectation de personnel et de ressources pour évaluer les voyageurs et les animaux malades, en prendre soin et les transporter, ainsi que la capacité d'inspecter les moyens de transport et les terminaux pour détecter les risques pour la santé publique. De plus, les points d'entrée canadiens désignés en vertu du RSI 
mènent systématiquement des exercices pour évaluer leur capacité d'intervention et s'assurer qu'ils satisfont toujours aux exigences du RSI (2005).

\section{Avant l'arrivée au Canada}

Avant d'entrer en eaux canadiennes, tous les navires de croisière internationaux doivent déclarer au Programme du public voyageur de l'Agence les taux de maladies gastro-intestinales (GI) parmi les passagers et les membres d'équipage. Une déclaration immédiate est exigée si le taux de maladies gastrointestinales dépasse les seuils clés en matière de santé publique. L'Agence collabore avec l'exploitant du navire de croisière afin d'atténuer les risques pour les autres passagers et membres d'équipage et s'assure que le navire a mis en place les procédures prévues pour la prévention des éclosions. Les agents d'hygiène du milieu de l'Agence, qui sont des inspecteurs certifiés en santé publique, peuvent mener une enquête à bord.

De même, les responsables d'avions doivent signaler à l'autorité aéroportuaire toute maladie ou tout décès survenus à bord, avant leur arrivée. Ces cas sont ensuite évalués par les agents de quarantaine de l'Agence, qui sont des infirmiers autorisés (3).

\section{À l'arrivée au Canada}

Tous les voyageurs qui arrivent au Canada doivent se présenter à un agent de l'Agence des services frontaliers du Canada, qui contrôle les voyageurs à la recherche de tout signe de maladie et leur demande s'ils ont en leur possession des produits alimentaires, végétaux ou animaux. Comme le prévoit la Loi sur la mise en quarantaine, tout voyageur qui obtient un résultat positif au test de dépistage d'une maladie transmissible potentielle posant un risque pour la santé publique est dirigé vers un agent de quarantaine pour subir une évaluation plus en profondeur, laquelle inclut la vérification des antécédents et de la température (3). Des agents de quarantaine sont en poste dans certains aéroports du pays qui reçoivent un volume élevé de voyageurs internationaux. Si le voyageur malade arrive à l'un de ces aéroports, l'évaluation est menée en personne. Si le voyageur malade se présente à un point d'entrée autre que ces aéroports, par exemple un port maritime ou un poste frontalier terrestre, l'évaluation est faite par téléphone.

Si l'agent de quarantaine soupçonne le voyageur d'être atteint d'une maladie transmissible qui présente un risque pour la santé publique, certaines mesures peuvent être prises. II peut par exemple ordonner au voyageur de se présenter à un hôpital situé à proximité pour subir une évaluation plus approfondie ou de se présenter à l'autorité locale de santé publique dans un délai précis pour faire l'objet d'un suivi. L'agent de quarantaine coordonnera avec l'autorité locale de santé publique, les services d'urgence et l'hôpital la prise en charge du voyageur malade.

Les agents d'hygiène du milieu interviennent également à bord des moyens de transport, en cas d'incidents préoccupants liés à la salubrité de l'eau ou des aliments ou aux conditions d'hygiène. Ces agents peuvent par exemple conseiller les préposés à l'entretien des avions sur la façon de nettoyer des déversements de liquides organiques ou faire enquête sur des rapports indiquant la présence de vecteurs tels que des rats à bord d'un navire.

\section{Après l'arrivée au Canada}

L'Agence fournit un soutien financier au réseau canadien de médecine des voyages (CanTravNet), un réseau d'experts cliniciens canadiens de la médecine des voyages et de la médecine tropicale (19). Les sites de CanTravNet font partie du réseau de surveillance mondiale GeoSentinel, un réseau international qui recueille des données sur les voyageurs qui reviennent au pays et les immigrants, et qui constitue également un réseau sentinelle pour les infections mondiales émergentes.

Lorsqu'une infection grave ou une éclosion est constatée, l'Agence a les ressources nécessaires pour déployer une équipe d'intervention rapide afin d'aider les autorités sanitaires locales, provinciales ou territoriales à protéger les travailleurs de la santé ainsi qu'à traiter la maladie et à en limiter la propagation (18). En vertu du RSI (2005), des renseignements sur certains événements de santé publique, notamment les maladies transmissibles, peuvent être communiqués à l'OMS pour faciliter la collaboration internationale durant une intervention de santé publique $(17,18)$. 


\section{Discussion}

L'Agence a mis en place une solide stratégie fédérale en matière de santé publique dans le but de réduire au minimum l'introduction des maladies transmissibles au Canada, en mettant l'accent sur la promotion de la santé, la prévention, la détection précoce et la rapidité d'intervention. L'Agence remplit son mandat en travaillant en étroite collaboration avec les prestataires de soins cliniques et les autorités de santé publique du niveau local à international ainsi qu'avec d'autres ministères fédéraux, dont l'Agence des services frontaliers du Canada et l'Agence canadienne d'inspection des aliments.

L'un des volets de la stratégie de l'Agence porte sur le contrôle des voyageurs aux postes frontaliers. Le contrôle des voyageurs s'appuie sur les signes et les symptômes observés, plutôt que sur des analyses de laboratoire. À leur arrivée, les voyageurs peuvent être asymptomatiques, soit parce qu'ils sont en période d'incubation ou qu'ils ont une infection subclinique, ne présenter que des symptômes légers, prendre des antipyrétiques pour réduire la fièvre ou ne pas déclarer leurs symptômes. Comme certains cas peuvent passer inaperçus, le dépistage à l'entrée a été décrit comme un moyen inefficace et exigeant en termes de ressources (13,20-22). L'efficacité du contrôle à la frontière est toutefois difficile à évaluer (23). Une étude a montré que le dépistage à l'entrée peut retarder la transmission locale d'une nouvelle souche grippale d'une à deux semaines, un délai qui pourrait être utilisé pour mieux préparer la communauté à une épidémie (21). De plus, la mise en œuvre de mesures visibles de dépistage et de contrôle à la frontière peut accroître la confiance, la sensibilisation et la conformité du public. Bien que le dépistage des voyageurs aux frontières comporte certaines limites, ce n'est qu'un des volets de la stratégie de l'Agence pour réduire au minimum l'introduction des maladies transmissibles au Canada.

De nouvelles menaces liées à des maladies infectieuses continueront d'apparaître dans le contexte de la mondialisation et de l'augmentation des voyages; un système intégré a toutefois été mis en place pour réduire au minimum l'introduction de ces maladies à la frontière et protéger et promouvoir la sécurité sanitaire à l'échelle nationale. L'Agence évalue et revoit régulièrement sa stratégie. Les progrès récents dans l'établissement d'une loi sur les pathogènes humains (2) et la mise sur pied d'équipes d'intervention rapide (10) sont des exemples des moyens mis en place par l'Agence à l'échelle nationale pour sans cesse consolider sa capacité à collaborer avec les autres dans la lutte contre les maladies infectieuses.

\section{Conclusion}

De nombreux ministères fédéraux collaborent aux postes frontaliers canadiens pour évaluer les personnes, les animaux, les aliments, les cargaisons, les bagages, les moyens de transport, les conteneurs, les marchandises et les colis et éviter que des infections, des toxines, des vecteurs et de possibles réservoirs de maladies infectieuses n'entrent au Canada. L'Agence continuera à proposer et à promouvoir des activités de promotion de la santé et de prévention, comme les alertes de santé des voyageurs et les recommandations en matière de santé des voyageurs à l'intention des cliniciens, ainsi qu'à participer aux inspections et aux activités de surveillance et d'intervention pour réduire au minimum l'introduction des maladies transmissibles au Canada.

\section{Remerciements}

Nous tenons à remercier toutes les personnes qui travaillent à ces programmes pour aider à garder les Canadiens en sécurité.

\section{Conflit d'intérêts}

Aucun

\section{Financement}

Le financement de ces programmes fédéraux provient du gouvernement du Canada. 


\section{Références}

(1) The World Bank. International tourism, number of arrivals. Washington DC: The World Bank; 2015. http://data.worldbank.org/indicator/ST.INT.ARVL/countries/1W?display=graph.

(2) Government of Canada. Human Pathogens and Toxins Regulations (HPTR). SOR/2015-44 October $27,2015$. (Disponible en français : http://gazette.gc.ca/rp-pr/p2/2015/2015-03-11/html/sor-dors44-fra.php).

(3) Government of Canada. Quarantine Act (S.C. 2005, c.20). (Disponible enfrançais http://laws.justice.gc.ca/fra/lois/Q-1.1/).

(4) Government of Canada. Potable Water Regulations for Common Carriers (C.R.C., c. 1105). (Disponible en français : http://laws-lois.justice.gc.ca/fra/reglements/C.R.C.\%2C_ch._1105/).

(5) Government of Canada. Food and Drugs Act (R.S.C., 1985, c. F-27). 20.

(Disponible en français : http://laws-lois.justice.gc.ca/fra/lois/F-27/)

(6) Government of Canada. Health of Animals Act (S.C. 1990, c.21).

(Disponible en français : http://laws-lois.justice.gc.ca/fra/lois/H-3.3/)

(7) Government of Canada. Plant Protection Act (S.C. 1990, c. 22).

(Disponible en français : http://laws-lois.justice.gc.ca/fra/lois/P-14.8/)

(8) Government of Canada. Immigration and Refugee Protection Act (S.C. 2001, c. 27).

(Disponible en français : http://laws-lois.justice.gc.ca/fra/lois/i-2.5/index.html)

(9) Labrie C, Lecordier S. Regulatory oversight of human pathogens and toxins in Canada. CCDR. 2015 Dec 17;41(S6):9-13. (Disponible en français : http://phac-aspc.gc.ca/publicat/ccdr-rmtc/15vol41/dr-rm41s6/regulations-reglementation-fra.php)

(10) Lior LY, Njoo H. Ready to Go! Canada's new Rapid Response Team. CCDR. 2015 Dec 17;41(S6):14-18. (Disponible en français : http://phac-aspc.gc.ca/publicat/ccdr-rmtc/15vol41/dr-rm41s-6/implementation-sciencefra.php)

(11) Public Health Agency of Canada. Travel health notices. Ottawa ON: PHAC; 2015. (Disponible en français : http://www.phac-aspc.gc.ca/tmp-pmv/index-fra.php).

(12) Memish ZA. The Hajj: Communicable and non-communicable health hazards and current guidance for pilgrims. Euro Surveill. 2010;15(39):pii=19671. http://www.eurosurveillance.org/ViewArticle.aspx?Articleld=19671.

(13) McCarthy A. Statement on Meningococcal Disease and the International Traveller. Can Commun Dis Rep. 2015 May;41(5):100-107. (Disponible en français : http://www.phac-aspc.gc.ca/publicat/ccdr-rmtc/15vol41/drrm41-05/com-2-fra.php).

(14) St. John RK, King A, de Jong D, Bodie-Collins M, Squires SG, Tam TWS. Border screening for SARS. Emerg Infect Dis. 2005 Jan;11(1). http://wwwnc.cdc.gov/eid/article/11/1/04-0835.

(15) Public Health Agency of Canada. About CATMAT. Ottawa ON: PHAC; 2015. (Disponible en français : http://www.phac-aspc.gc.ca/tmp-pmv/catmat-ccmtmv/index-fra.php).

(16) Health Canada. Cruise ship inspection program. Ottawa ON: Health Canada; 2011. (Disponible en français : http://www.hc-sc.gc.ca/hl-vs/travel-voyage/general/ship-navire-fra.php).

(17) World Health Organization. International Health Regulations 2005. Geneva: WHO; 2005. (Disponible en français : http://www.who.int/ihr/fr/).

(18) Public Health Agency of Canada. International Health Regulations (IHR) (2005). Ottawa ON: PHAC; 2014. (Disponible en français : http://www.phac-aspc.gc.ca/ep-mu/ihr-rsi/index-fra.php).

(19) International Society of Travel Medicine (ISTM). Canadian Travel Medicine Network of the International Society of Travel Medicine. Dunwoody GA: ISTM; 2015. http://www.istm.org/cantravnet.

(20) Selvey LA, Antão C, Hall R. Evaluation of border entry screening for infectious diseases in humans. Emerg Infect Dis. 2015 Feb;21(2). http://dx.doi.org/10.3201/eid2102.131610.

(21) Cowling BJ et al. Entry screening to delay local transmission of pandemic influenza A (H1N1). BMC Infect. Dis. 2010;10:82.

(22) Priest P, Jennings L, Duncan A, Brunton C, Baker M. Effectiveness of border screening for detecting influenza in arriving airline travelers. Am J Public Health. 2015 October;105(Suppl 4):S607-S613.

(23) World Health Organization. Public health measures taken at international borders during early stages of pandemic influenza A (H1N1) 2009: preliminary results. Wkly Epidemiol Rec. 2010;85:186-95. 